\title{
Orbitofrontal or accumbens dopamine depletion does not affect risk-based decision making in rats
}

\author{
Bettina Mai $^{1}$ - Wolfgang Hauber ${ }^{1}$
}

Published online: 10 April 2015

(C) Psychonomic Society, Inc. 2015

\begin{abstract}
Considerable evidence has implicated dopamine (DA) signals in target regions of midbrain DA neurons such as the medial prefrontal cortex or the core region of the nucleus accumbens in controlling risk-based decision-making. However, to date little is known about the contribution of DA in the orbitofrontal cortex (OFC) and the medial shell region of the nucleus accumbens (AcbS) to risk-based decision-making. Here we examined in rats the effects of 6hydroxydopamine-induced DA depletions of the OFC and AcbS on risky choice using an instrumental two-lever choice task that requires the assessment of fixed within-session reward probabilities that were shifted across subsequent sessions, i.e., rats had to choose between two levers, a small/ certain lever that delivered one certain food reward (one pellet at $p=1$ ) and a large/risky lever that delivered a larger uncertain food reward with decreasing probabilities across subsequent sessions (four pellets at $\mathrm{p}=0.75,0.5,0.25,0.125$, 0.0625 ). Results show that systemic administration of amphetamine or cocaine increased the preference for the large/risky lever. Results further demonstrate that, like sham controls, rats with OFC or AcbS DA depletion were sensitive to changes in probabilities for obtaining the large/risky reward across sessions and displayed probabilistic discounting. These findings point to the view that the basal capacity to evaluate the magnitude and likelihood of rewards associated with alternative courses of action as well as long-term changes of reward probabilities does not rely on DA input to the AcbS or OFC.
\end{abstract}

Wolfgang Hauber

hauber@bio.uni-stuttgart.de

1 Department of Animal Physiology, University of Stuttgart, Pfaffenwaldring 57, 70550 Stuttgart, Germany
Keywords Decision-making · Risk · Orbitofrontal cortex · Nucleus accumbens $\cdot$ Amphetamine $\cdot$ Cocaine $\cdot$ Dopamine Rat

\section{Introduction}

Considerable evidence suggests that brain dopamine (DA) transmission supports risk-based decision-making. For instance, midbrain DA neurons are activated by stimuli predicting risky rewards and generate a neuronal signal that varies monotonically with risk (Fiorillo, Tobler, \& Schultz, 2003). Furthermore, systemic D1- and/or D2-receptor blockade markedly reduced the preference for large/risky rewards suggesting that decreased DA transmission can reduce risky choice (St Onge \& Floresco, 2009). Moreover, drug-induced manipulations of DA transmission altered risk-based decisionmaking, e.g., a history of alcohol intake in adolescent rats compromised DA signaling to risk and increased risk preference (Nasrallah et al., 2011).

DA signaling in two terminal regions of midbrain DA neurons, i.e., the medial prefrontal cortex ( $\mathrm{mPFC}$ ) and the nucleus accumbens (Acb), plays a crucial role in risk-based decisionmaking. Pharmacological studies revealed that intra-mPFC D1-receptor blockade reduced, whereas intra-mPFC D2-receptor blockade increased, risk-taking, suggesting that mPFC DA receptors contribute to risk-based decision-making in a dissociable manner (St Onge, Abhari, \& Floresco, 2011). Studies using fast-scan cyclic voltammetry further indicated that phasic DA release in the core subregion of the Acb (AcbC) encodes reward value, a signal that could influence decisions to take risks (Sugam, Day, Wightman, \& Carelli, 2012). Also, microdialysis studies recently revealed that slow fluctuations of $\mathrm{mPFC}$ and Acb DA release are associated with 
different aspects of risk-based decision-making (St Onge, Ahn, Phillips, \& Floresco, 2012).

Of note, the effects of experimental manipulations of DA transmission on risk-based decision-making critically depend on the task employed. For instance, DA in the mPFC and Acb plays a crucial role in risk-based decision-making in complex instrumental tasks that demand an assessment of short-term within-session changes in reward probabilities associated with different levers (St Onge, et al., 2011; Stopper, Khayambashi, \& Floresco, 2013). By contrast, DA in the mPFC and AcbC does not support risk-based decision-making in simpler instrumental choice tasks involving fixed within-session reward probabilities that were shifted across subsequent sessions (Mai \& Hauber, 2012).

The orbitofrontal cortex (OFC) (O'Neill \& Schultz, 2010; Roitman \& Roitman, 2010) and the shell region of the Acb (AcbS) (Stopper \& Floresco, 2011) are two other terminal regions of midbrain DA neurons and key structures of the neural network that governs risk-based decision-making. However, to date little is known about the contribution of DA in these two regions to risk-based decision-making. Therefore, this study sought to examine the effects of OFC and AcbS DA depletions on risky choice. If DA risk signals to these regions support the evaluation of reward risk, we hypothesize that animals subjected to DA depletions in the OFC or AcbS are impaired in assessing the likelihood of rewards in risky choice tasks. We used a simple instrumental two-lever choice task in which rats had to choose between two levers, a small/certain lever that delivered one certain food reward and a large/risky lever that delivered a larger uncertain food reward with decreasing probabilities across subsequent sessions. To assess the sensitivity of this task to experimental manipulations of brain DA transmission, we also examined the effects of systemic administration of amphetamine and cocaine.

\section{Methods}

All animal experiments were performed according to the German Law on Animal Protection and approved by the proper authorities.

\section{Experiment 1}

Across experiments 1-3, rats were examined in a probabilistic choice task in which rats chose between two levers, a small/ certain lever that delivered one food pellet at $\mathrm{p}=1$ and a large/ risky lever that delivered foour food pellets with decreasing probabilities ( $p=0.75,0.5,0.25,0.125,0.0625)$ across five subsequent test days (one test session per day). This task is referred to here as probabilistic discounting task ("pDISCtask"). In experiment 1 , we examined whether this task is sensitive to systemic manipulations of DA transmission by amphetamine and cocaine.

\section{Subjects}

Male Lister hooded rats (Charles River, Sulzfeld, Germany) weighing between 200 and 225 g upon arrival were used. They were housed in groups of up to four animals in transparent plastic cages $(60 \mathrm{~cm} \times 38 \mathrm{~cm} \times 20 \mathrm{~cm}$; Tecniplast, Milan, Italy) in a 12-h:12-h light-dark cycle (lights on at 7.00 a.m.) with ad libitum access to water. Upon arrival standard laboratory chow (Altromin, Lage, Germany) was given ad libitum for at least 5 days Thereafter, food was restricted to $15 \mathrm{~g}$ per animal per day to maintain them on approximately $85 \%$ of their free-feeding weight. For environmental enrichment, a plastic tube (length $20 \mathrm{~cm}$, diameter $12 \mathrm{~cm}$ ) was fixed on the lid of each cage. Temperature $\left(22 \pm 2{ }^{\circ} \mathrm{C}\right)$ and humidity $(50 \pm$ $10 \%$ ) were kept constant in the animal house.

\section{Apparatus}

Training and testing took place in identical operant chambers $(24 \times 21 \times 30 \mathrm{~cm}$; Med Associates, St. Albans, VT, USA) housed within sound-attenuating cubicles. Each operant chamber contained a central food magazine that was positioned in the right wall. Casein pellets (45-mg dustless precision pellets ${ }^{\circledR}$; Bioserv, Frenchtown, NJ, USA) were delivered by a pellet dispenser to the food magazine. The food magazine was equipped with an infrared head entry detector and a stimulus light was installed above the food magazine. Two retractable levers were on either side of the food magazine. A house light mounted on the top center of the opposite wall illuminated the chamber. A computer with the PC program MED-PC IV (MedPCSoftware; Med Associates) controlled the equipment and recorded the data.

\section{Habituation and lever-press training}

On the first day, all rats were habituated to the operant chamber on one day in a 15-min session with free access to 25 pellets on the food magazine. For the entire session, the chamber was illuminated by the house and stimulus light. On the next day, rats received a $30-$ min session with pellet delivery after a variable interval (average interval $60 \mathrm{~s}$, minimum interval $10 \mathrm{~s}$; maximum interval $110 \mathrm{~s}$ ) and pellet delivery was paired with illumination of the stimulus light. Thereafter, animals were trained for 2 days in two daily 15-min sessions to lever press under a fixed ratio-1 (FR-1) schedule for each lever. A lever was extended for the entire session and the house light always illuminated. Lever press training was followed by training on a simplified version of the full task for 3 days with one daily session. The task comprised 48 training trials per session; each trial started with house and 
stimulus light illumination and with the levers retracted. A nosepoke response in the food magazine within $10 \mathrm{~s}$ extinguished the stimulus light and extended one of the levers. The order of lever presentation was chosen pseudo-randomly, but each lever was presented in 24 trials. If the rat responded to the lever within $10 \mathrm{~s}$, the lever retracted and a single pellet was delivered and the house light extinguished. The inter-trial interval (ITI) was set at $35 \mathrm{~s}$. Failure to respond within $10 \mathrm{~s}$ to start the trial with a nosepoke response or to lever press resulted in a time-out period of $35 \mathrm{~s}$ during which all lights extinguished and levers retracted. These trials were recorded as omissions. Animals were removed once all trials were completed or after $60 \mathrm{~min}$ had elapsed.

\section{Choice task with probabilistic discounting (pDISC-task)}

The pDISC-task used here was based on protocols described by Cardinal and Howes (2005) and Nasrallah, Yang, and Bernstein (2009) (Fig. 1). For all testing sessions one of the two levers was designated as being the small/certain lever (1 pellet, $\mathrm{p}=1$ ) and the other as the large/risky lever (four pellets with decreasing odds; $p=0.75,0.5,0.25,0.125,0.0625$,

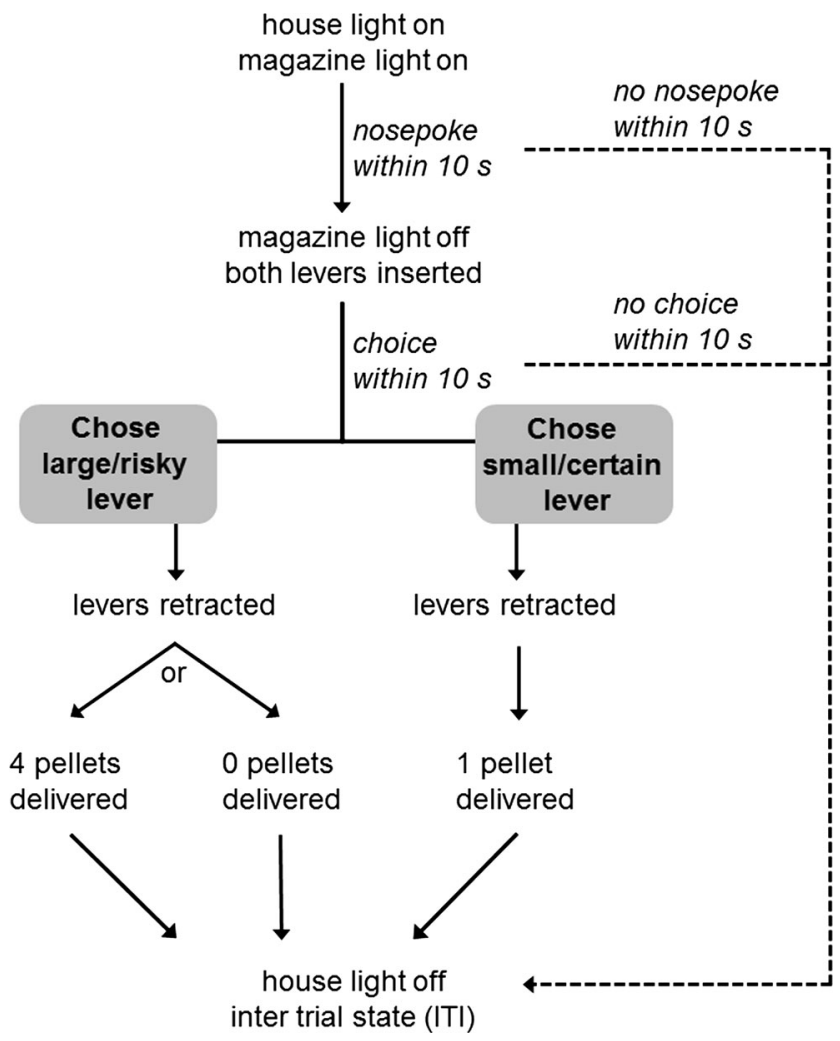

Fig. 1 Schematic representation of a single free-choice trial in the probabilistic discounting task: Choice of the small/certain lever led to a certain delivery of one pellet and choice of the large/risky lever led to a delivery of four pellets. The probability of delivery of the large/risky lever was declined systematically across sessions from $p=0.75,0.5,0.25$, $0.125,0.0625$, respectively respectively). This assignment was kept constant for each rat throughout the study and was counterbalanced across rats. After choosing the certain lever a small reward (one pellet, $p$ $=1$ ) was delivered, after choosing the risky lever a large reward (four pellets with different odds) was delivered. The odds of obtaining a risk/large reward were kept constant within a session but were reduced across five subsequent sessions.

Each session in the pDISC-task consisted of 32 forced trials followed by 24 free-choice trials. At the beginning of each trial, house and stimulus lights were illuminated, and levers remained retracted. Upon a nosepoke response to the food magazine within $10 \mathrm{~s}$, the stimulus light extinguished. In forced trials, one of the levers was extended in order to expose a rat to the current response option and its associated value. Each lever was presented in 16 forced trials with the order of presentation chosen pseudo-randomly. To examine choice behavior of rats, in free-choice trials both levers were extended at the same time. After a response to a lever in forced or choice trials within $10 \mathrm{~s}$, all levers were retracted and a reward was delivered with the associated probability. Multiple pellets were delivered $0.5 \mathrm{~s}$ apart without any prior cue. After pellet delivery, the house light extinguished to mark the end of a trial. Then the inter-trial interval (ITI), which was set at $35 \mathrm{~s}$, started. Failure to respond within $10 \mathrm{~s}$ to start the trial with a nosepoke response or to lever press resulted in a time-out period of $35 \mathrm{~s}$ during which all lights were extinguished and levers retracted. These trials were recorded as trial omissions. Rats were removed once all trials were completed or after $60 \mathrm{~min}$ had elapsed. Testing of rats on the pDISC-task was performed upon completion of training on the simplified task version (see above). Testing on the pDISC-task was performed across subsequent blocks; one block always consisted of five consecutive testing days, on each testing day the odds of obtaining the large/risky reward were constant but declined across subsequent days $(\mathrm{p}=0.75,0.5,0.25,0.125,0.0625$, respectively).

\section{Effects of d-amphetamine and cocaine on risk-based decision-making}

Rats ( $\mathrm{n}=20$ ) were tested across four subsequent blocks each consisting of five testing days as described above. On block 1, task performance of rats was assessed without drug administration; rats were assigned to two treatment groups matched on the basis of choice behavior during this block. On block 2 one treatment group received vehicle $(0.9 \%$ saline, $1 \mathrm{ml} / \mathrm{kg}$, intraperitoneally (i.p.)), the other $d$-amphetamine (Sigma Aldrich, Steinheim, Germany, $2 \mathrm{mg} / \mathrm{kg}$, i.p.) $30 \mathrm{~min}$ prior to testing. On block 3 animals received re-training without drug treatment. On block 4 , one group received vehicle $(0.9 \%$ saline, $1 \mathrm{ml} / \mathrm{kg}$, i.p.), the other cocaine (Sigma Aldrich, Steinheim, Germany, $15 \mathrm{mg} / \mathrm{kg}$, i.p.) $15 \mathrm{~min}$ prior to testing using a cross-over design, i.e. the treatment group that received vehicle in block 
2 was given cocaine in block 4 , the treatment group that received amphetamine in block 2 was given vehicle in block 4 .

The dose of amphetamine $(2 \mathrm{mg} / \mathrm{kg}$, i.p.) was chosen according to our pilot studies, lower doses used in other risk tasks in rats that employed a within-subject design (Simon, Gilbert, Mayse, Bizon, \& Setlow, 2009; St Onge \& Floresco, 2009) had only moderate effects in our task that employed an in-between subject design. The dose for cocaine $(15 \mathrm{mg} / \mathrm{kg}$, i.p.) was chosen because this dose produced equipotent effects on lever pressing to the dose of amphetamine used here (Poncelet, Chermat, Soubrie, \& Simon, 1983).

\section{Data analysis and statistics}

In choice trials, large/risky and small/certain lever presses were recorded. In addition, response latencies for the large/ risky lever in forced trials as well as trial omissions in forced and choice trials were measured. Percentage choices of the large/risky reward lever, response latencies and trial omissions are given as means \pm standard error of the mean (SEM). The data were subjected to a repeated-measures analysis of variance (ANOVA) with two within-subject factors (days of testing, large reward probability condition) and one betweensubjects factor (treatment). All statistical computations were carried out with STATISTICA ${ }^{\mathrm{TM}}$ (version 7.1, StatSoft ${ }^{\circledR}$, Inc., Tulsa, OK, USA). The level of statistical significance $(\alpha-$ level) was set at $\mathrm{p} \leq 0.05$ ( $\alpha$-levels $>0.05$ were designated as not significant (n.s.)).

\section{Experiment 2}

Here we examined the effects of DA depletion within the medial AcbS on risk-based decision-making in the pDISCtask. Unless otherwise noted, the same procedures as in Experiment 1 were used.

\section{Training}

Animals were trained on the pDISC-task and tested in one block, i.e. five subsequent daily sessions with the odds for the large risky reward decreasing across sessions. Rats were assigned to treatment groups matched on the basis of their choice behavior during the first block and received either 6hydroxydopamine (6-OHDA) lesions or sham lesions of the AcbS.

\section{Surgery}

For stereotaxic surgery, the animals were anesthetized with sodium pentobarbital $(60 \mathrm{mg} / \mathrm{kg}$, i.p.; Narcoren, Merial, Hallbergmoos, Germany) and xylazine (4 mg/kg, intramuscularly (i.m.); Rompun, Bayer, Leverkusen, Germany) and secured in a stereotaxic apparatus with atraumatic ear bars (Kopf
Instruments, Tujunga, CA, USA). The skull surface was exposed and small holes were drilled bilaterally above the target region. Animals subjected to AcbS DA depletion received two injections per hemisphere of $6 \mu \mathrm{g}$ 6-OHDA hydrochlorid (Sigma-Aldrich, Steinheim, Germany) in saline containing $0.01 \%$ ascorbic acid (Sigma-Aldrich, Steinheim, Germany) in a volume of $0.25 \mu \mathrm{l}$ using a $1 \mu \mathrm{l}$ Hamilton syringe with the tooth bar set at $-3.3 \mathrm{~mm}$ below the interaural line (coordinates: $\mathrm{AP}+1.3 \mathrm{~mm}$; $\mathrm{ML} \pm 0.9 \mathrm{~mm}$; $\mathrm{DV}-7.2 \mathrm{~mm}$ and $-7.6 \mathrm{~mm})$. Sham controls received two injections per hemisphere of $0.25 \mu \mathrm{l}$ saline containing $0.01 \%$ ascorbic acid. Infusion time was $2 \mathrm{~min}$ and the needle was left in place to allow diffusion for $5 \mathrm{~min}$. After surgery, rats were sutured and received a subcutaneous injection of $2 \mathrm{ml}$ saline and an analgesic drug (Rimadyl, Pfizer, Karlsruhe, Germany; $4 \mathrm{mg} / \mathrm{kg}$ ). Animals were allowed to recover for 10-14 days before the onset of behavioral testing.

\section{Behavioral procedures}

Post-lesion, animals were tested on the pDISC-task across five consecutive testing days, on each testing day the odds of obtaining the large/risky reward was constant but declined across subsequent days $(p=0.75,0.5,0.25,0.125,0.0625$, respectively). Results indicated that rats with AcbS DA depletion seemed to have a reduced preference for the large reward/ risky lever in the first session with $p=0.75$. To investigate this possibility in more detail, upon completion of the pDISC task, we trained both treatment groups subsequently in a simpler task variant with a fixed probability for the large reward (termed here as choice task with fixed probabilities, "pFIXtask"), i.e., rats chose between small/certain lever that delivered one single food pellet at $p=1$ and a large/risky lever that delivered four food pellets at $p=0.75$ over four consecutive testing days (one test session per day).

Furthermore, we performed an additional study with a separate cohort of naïve male Lister hooded rats $(n=13)$ to test for the sensitivity of the pFIX-task to reduced DA activity induced by systemic administration of flupenthixol. Animals were first trained on the pDISC task as described above and, subsequently, on the pFIX task with the probability of obtaining the large/risky reward set at $75 \%$ for several days. Thereafter, animals were assigned to two treatment groups that received 60 min prior behavioral testing either saline ( $1 \mathrm{ml} / \mathrm{kg}$, i.p.) or the mixed D1/D2 receptor antagonist cis-(z)-flupenthixol dihydrochloride (Sigma Aldrich) $(0.25 \mathrm{mg} / \mathrm{kg}$, i.p. $)$ dissolved in saline. Drug effects were assessed in one session on a single test day.

\section{Histology}

Tyrosine hydroxylase $(\mathrm{TH})$ immunohistochemistry was used to assess the exact location and extent of the loss of DA 
terminals. On completion of the behavioral testing, animals were euthanized by an overdose of isoflurane (Abbott, Wiesbaden, Germany) and perfused transcardially with $0.01 \%$ heparin sodium salt in phosphate-buffered saline (PBS) followed by $4 \%$ paraformaldehyde in PBS. The brains were extracted, post-fixed in paraformaldehyde for $24 \mathrm{~h}$ and dehydrated in $30 \%$ sucrose for at least $48 \mathrm{~h}$. Coronal brain sections were cut (35-40 $\mu \mathrm{m}$; Microm, Walldorf, Germany). The slices were initially washed in TRIS-buffered saline (TBS; $3 \times 10 \mathrm{~min}$ ), treated for $15 \mathrm{~min}$ with TBS containing $2 \%$ hydrogen peroxide and $10 \%$ methanol, washed again in TBS $(3 \times 10 \mathrm{~min})$ and then blocked for 20 min with $4 \%$ natural horse serum (NHS; Vector Laboratories Burlingame, CA, USA) in TBS containing $0.2 \%$ Triton X-100 (TBS-T; Sigma Aldrich). Slices were incubated overnight at $4{ }^{\circ} \mathrm{C}$ in a primary antibody (mouse, anti-TH, 1:7500 in TBS-T containing $4 \%$ NHS; Immunostar, Hudson, WI, USA), then washed in TBS-T $(3 \times 10 \mathrm{~min})$ and incubated in a secondary antibody (horse, anti-mouse, rat-adsorbed, biotinylated $\operatorname{IgG}(\mathrm{H}+\mathrm{L})$, 1:500 in TBS-T containing $4 \%$ NHS; Vector Laboratories) for $90 \mathrm{~min}$ at room temperature. Using the biotin-avidin system, slices were washed in TBS-T containing the avidin-biotinylated enzyme complex (1:500, ABC-Elite Kit; Vector Laboratories) for $60 \mathrm{~min}$ at room temperature, washed in TBS $(3 \times 10 \mathrm{~min})$ and stained with 3,3'-diaminobenzidine (DAB Substrate Kit, Vector Laboratories). The brain slices were then washed in TBS $(3 \times 10 \mathrm{~min})$, mounted on coated slides, dried overnight, dehydrated in ascending alcohol concentrations, treated with xylene and finally coverslipped using DePex (Serva, Heidelberg, Germany). To determine the size and placement of the lesions the TH-immunoreactivity was analyzed with reference to Paxinos and Watson's atlas (1998). We performed a qualitative reconstruction of lesioned prefrontal or accumbens regions which, relative to sham controls, displayed lower TH-immunoreactivity, i.e., reduced $\mathrm{TH}$ staining (see Fig. 3b).

\section{Experiment 3}

Here we examined the effects of OFC DA depletion on riskbased decision-making in the pDISC-task and subsequently in the pFIX-task. Unless otherwise noted, the same procedures as in Experiment 2 were used.

\section{Surgery}

For DA depletion of the OFC, rats received 6-OHDA ( $8 \mu \mathrm{g} /$ $\mu \mathrm{l}$ ) in saline containing $0.01 \%$ ascorbic acid at the following coordinates: $0.2 \mu \mathrm{l}$ at $\mathrm{AP}+4.0 \mathrm{~mm}$; $\mathrm{ML} \pm 0.8 \mathrm{~mm}$; DV $-4.6 \mathrm{~mm} ; 0.3 \mu \mathrm{l}$ at $\mathrm{AP}+3.7 \mathrm{~mm} ; \mathrm{ML} \pm 2.0 \mathrm{~mm}$; DV $-4.5 \mathrm{~mm} ; 0.3 \mu \mathrm{l}$ at $\mathrm{AP}+3.2 \mathrm{~mm}$; ML $\pm 2.6 \mathrm{~mm}$; DV $-5.1 \mathrm{~mm}$. Sham controls received injections of the same volume of saline containing $0.01 \%$ ascorbic acid at the same coordinates. Infusion time was $3 \mathrm{~min}$ and the needle was left in place to allow for a diffusion of $2 \mathrm{~min}$.

\section{Behavioral procedures}

Treatment groups were tested in the pDISC and, subsequently, the pFIX-task as described above.

\section{Results}

\section{Experiment 1}

Results showed that, in rats to be treated with drugs in subsequent blocks, the preference for the large/risky lever markedly declined across subsequent days with the odds obtaining a large reward becoming increasingly lower (Fig 2). An ANOVA revealed no main effect of treatment $(\mathrm{F}(1,18)=$ 0.05 , n.s. $)$, but a main effect of days $(\mathrm{F}(4,72)=37.92, \mathrm{p}<$ $0.001)$, and no days $\times$ treatment interaction $(\mathrm{F}(4,72)=0.19$, n.s.). By contrast, relative to vehicle controls, amphetaminetreated animals had an increased preference for the large/risky reward, but still display probabilistic discounting across days. Accordingly, an ANOVA revealed main effects of treatment $(\mathrm{F}(1,18)=7.09, \mathrm{p}<.05)$ and days $(\mathrm{F}(4,72)=22.63, \mathrm{p}<$ $0.001)$, but no day $\times$ treatment interaction $(\mathrm{F}(4,72)=1.12$, n.s). Likewise, relative to vehicle controls, the number of trial omissions was increased in amphetamine-treated animals (Table 1). An ANOVA revealed main effects of treatment $(\mathrm{F}(1,18)=15.90, \mathrm{p}<0.001)$, days $(\mathrm{F}(4,72)=3.25, \mathrm{p}<$ $0.05)$ and a day $\times$ treatment interaction $(\mathrm{F}(4,72)=3.24, \mathrm{p}<$ $0.05)$. By contrast, response latencies were not altered in amphetamine-treated animals (Table 1). An ANOVA showed no main effects of treatment $(\mathrm{F}(1,18)=2.43$, n.s. $)$, but of days $(\mathrm{F}(4,72)=4.76, \mathrm{p}<0.01)$ and no day $\times$ treatment interaction $(\mathrm{F}(4,72)=1.35$, n.s. $)$.

Cocaine-treated rats showed an increased preference for the large/risky lever if the odds of obtaining large reward were high. In line with this description, an ANOVA revealed no main effect of treatment $(\mathrm{F}(1,18)=1.78$, n.s $)$, but of days $(\mathrm{F}(4,72)=19.21, \mathrm{p}<0.001)$ and an almost significant day $\times$ treatment interaction $(F(4,72)=2.41, p=0.06)$. In addition, the number of trial omissions was increased in cocaine-treated animals (Table 1). An ANOVA revealed a main effect of treatment $(\mathrm{F}(1,18)=9.88, \mathrm{p}<0.01)$ but not of days $(\mathrm{F}(4,72)=$ 1.37 , n.s. $)$ and there was no day $\times$ treatment interaction $(\mathrm{F}(4$, $72)=1.24$, n.s.). Moreover, relative to vehicle controls, cocaine-treated animals had increased response latencies (Table 1). An ANOVA showed a main effect of treatment $(\mathrm{F}(1,18)=5.53, \mathrm{p}<0.05)$, and a near significant main effect of days $(\mathrm{F}(4,72)=2.31, \mathrm{p}=0.07)$ and a day $\times$ treatment interaction $(\mathrm{F}(4,72)=3.00, \mathrm{p}<0.05)$. 
Table 1 Mean ( \pm SEM) trial omissions and response latencies from all sessions and from sessions with decreasing reward probabilities - experiment 1

\begin{tabular}{|c|c|c|c|c|c|c|}
\hline large/risky lever probability (\%) & overall mean & $75 \%$ & $50 \%$ & $25 \%$ & $12.5 \%$ & $6.25 \%$ \\
\hline & vehicle & & & & & \\
\hline omissions (number of trials per session) & $0.6(0.4)$ & $0.0(0.0)$ & $0.5(0.2)$ & $0.3(0.2)$ & $0.2(0.1)$ & $2.2(1.5)$ \\
\hline \multirow[t]{2}{*}{ response latency (s) } & $1.2(0.2)$ & $1.2(0.2)$ & $1.1(0.1)$ & $1.0(0.2)$ & $1.2(0.2)$ & $1.6(0.4)$ \\
\hline & amphetamine & & & & & \\
\hline omissions (number of trials per session) & $10.1(3.3)^{* *}$ & $4.3(1.8)$ & $18.5(5.3)$ & $6.3(1.8)$ & $12.8(4.6)$ & $8.5(3.2)$ \\
\hline \multirow[t]{2}{*}{ response latency (s) } & $1.6(0.2)^{*}$ & $2.1(0.3)$ & $1.4(0.2)$ & $1.2(0.1)$ & $1.3(0.2)$ & $1.9(0.3)$ \\
\hline & vehicle & & & & & \\
\hline omissions (number of trials per session) & $0.0(0.0)$ & $0.0(0.0)$ & $0.0(0.0)$ & $0.1(0.1)$ & $0.0(0.0)$ & $0.0(0.0)$ \\
\hline \multirow[t]{2}{*}{ response latency (s) } & $0.8(0.1)$ & $0.9(0.1)$ & $0.8(0.1)$ & $0.8(0.0)$ & $0.7(0.1)$ & $0.9(0.1)$ \\
\hline & cocaine & & & & & \\
\hline omissions (number of trials per session) & $2.4(1.0) * *$ & $0.9(0.3)$ & $2.9(1.2)$ & $3.4(1.2)$ & $2.8(1.5)$ & $1.9(0.7)$ \\
\hline \multirow[t]{2}{*}{ response latency (s) } & $1.2(0.2)^{* *}$ & $0.9(0.1)$ & $1.2(0.2)$ & $1.1(0.2)$ & $1.2(0.2)$ & $1.4(0.2)$ \\
\hline & $\begin{array}{l}* \quad \mathrm{p}<0.05 \\
* * \mathrm{p}<0.001 \\
\text { vs. vehicle (t-test) }\end{array}$ & \multicolumn{5}{|c|}{$\# \mathrm{p}<0.05$ main effect of treatment (ANOVA) } \\
\hline
\end{tabular}

\section{Experiment 2}

The lesion size and placement were assessed by reconstructing the damaged areas on stereotaxic atlas templates from Paxinos and Watson (1998). No animal was excluded due to misplaced AcbS DA depletions. Final sample sizes were as follows: sham controls, $n=10$, rats with AcbS DA depletion, $n=14$. Figure 3a provides a schematic representation of the animals with the minimum and the maximum extent of AcbS lesion, respectively. Additionally, a photomicrograph of a representative 6-OHDA-induced lesion is shown in Fig. 3b. The analysis shows that TH-positive fibers in the AcbS were abundant in sham-lesioned rats but rare in rats with 6-OHDA lesions. In most animals, loss of TH-positive fibers in the AcbS appeared from 2.2 to $0.2 \mathrm{~mm}$ relative to bregma, with the maximum extension at approximately $1.5 \mathrm{~mm}$ relative to bregma. Furthermore, 6-OHDA lesions caused some damage to the medial part of the $\mathrm{AcbC}$ and the olfactory tubercle.

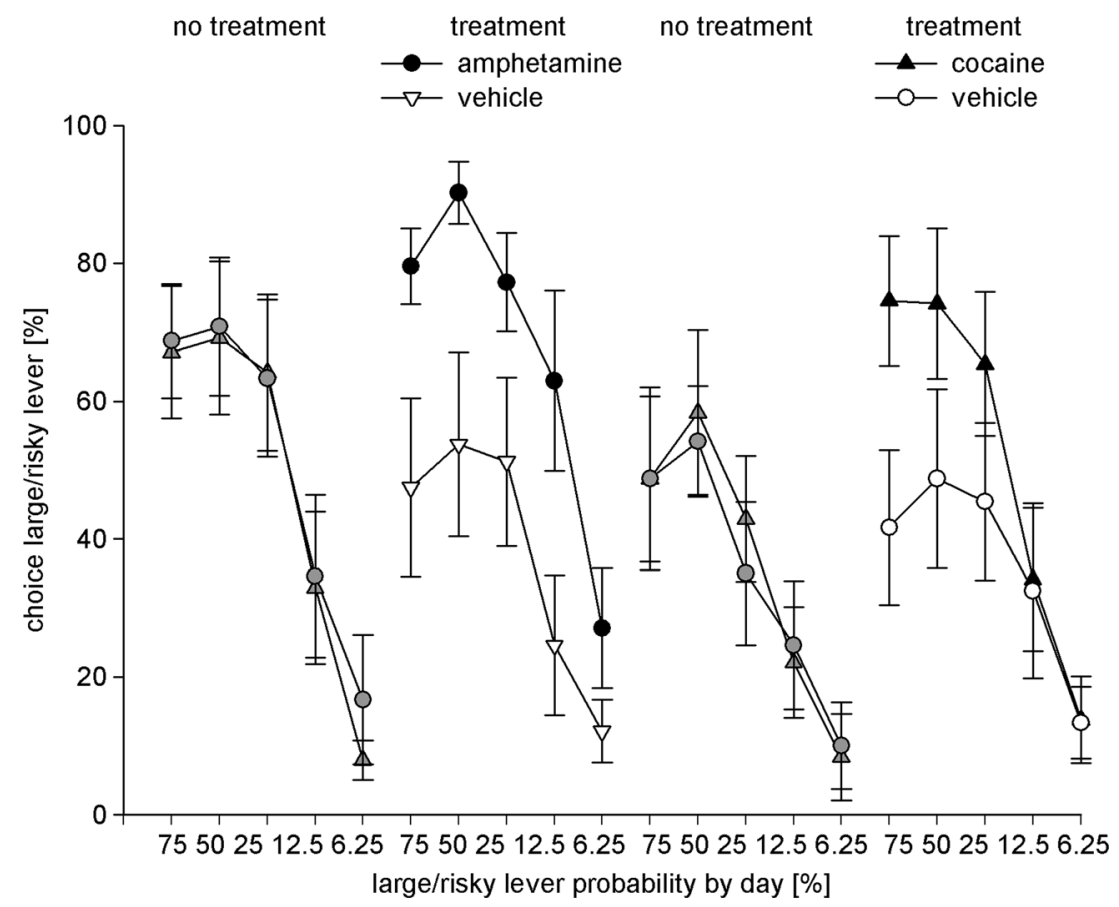

Fig. 2 Effects of amphetamine (2 mg/kg, intraperitoneally (i.p.)) and cocaine $(15 \mathrm{mg} / \mathrm{kg}$, i.p.) on probabilistic choice. Mean $( \pm$ SEM) percentages of large/risky lever choices per day are shown for both groups $(\mathrm{n}=10$, circles and $\mathrm{n}=10$, triangles). Amphetamine (black circles) and cocaine (black triangles) increased preference for the large/ risky reward. In blocks without drug treatment (grey symbols), the preference for the large/risky lever markedly declined across days but did not differ between the groups 


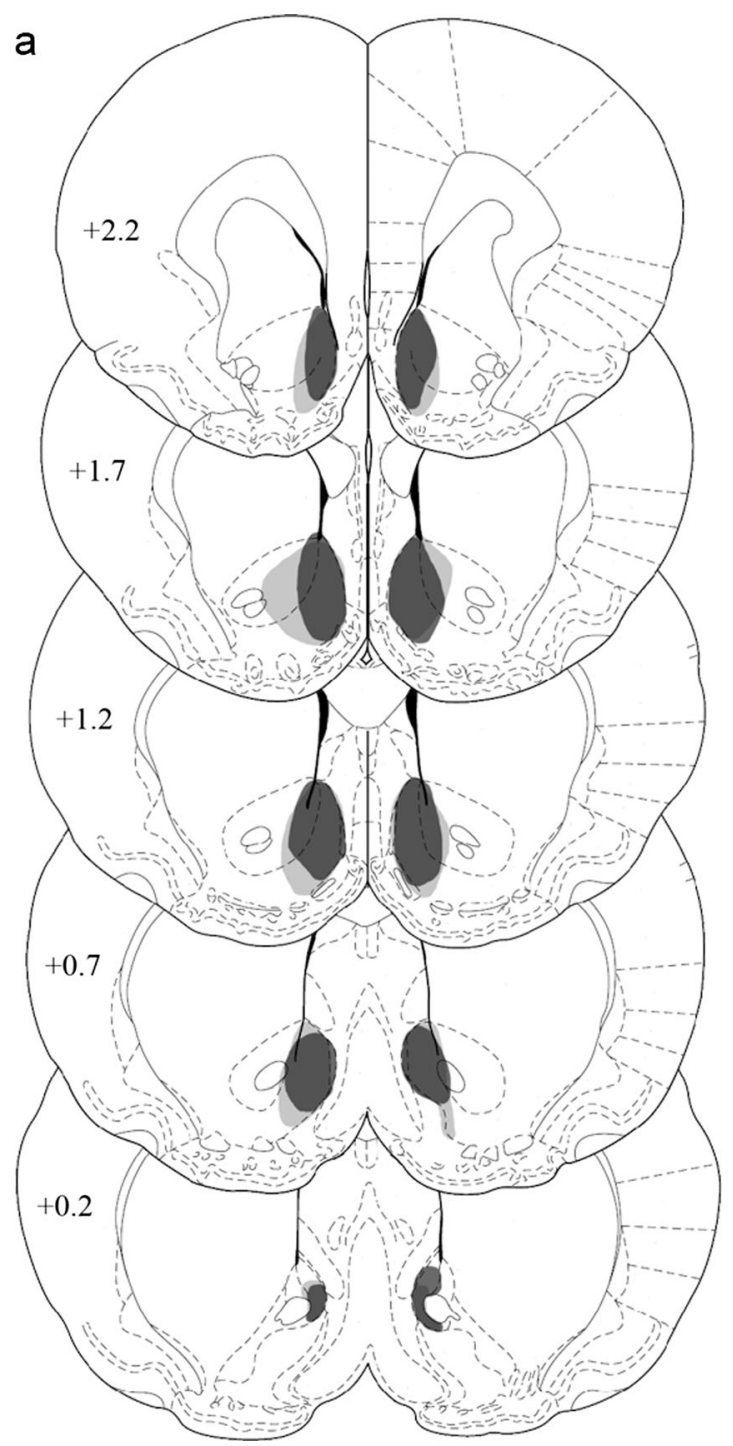

\section{b sham control}

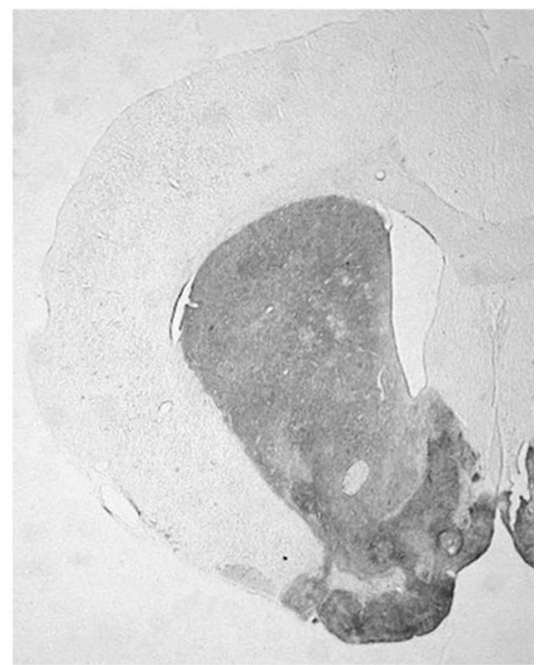

AcbS DA depletion

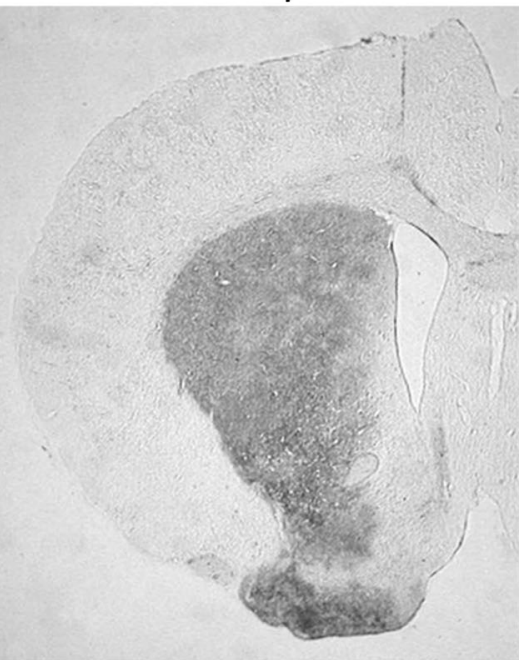

Fig. 3 (a) Loss of TH-positive fibers in the AcbS of 6-OHDA-lesioned rats. Qualitative reconstruction of the region with, relative to sham controls, reduced TH-staining, indicating in each slice the animal with the largest lesion (gray areas) and the animal with the smallest lesion (black areas). The numbers indicate the distances from bregma, in millimeters. (b) Representative photomicrograph of a 6-OHDA-induced loss of TH-immunoreactive fibers in the AcbS (top: AcbS sham lesion; bottom: AcbS DA depletion)

Table 2 Mean $( \pm$ SEM) trial omissions and response latencies from all sessions and from sessions with decreasing reward probabilities - experiment 2 and 3

\begin{tabular}{|c|c|c|c|c|c|c|c|c|c|c|c|}
\hline \multirow[b]{2}{*}{ large/risky lever probability (\%) } & \multirow[b]{2}{*}{ overall mean } & \multicolumn{5}{|c|}{ pDISC } & & \multicolumn{4}{|c|}{ pFIX } \\
\hline & & $75 \%$ & $50 \%$ & $25 \%$ & $12.5 \%$ & $6.25 \%$ & & $75 \%$ & $75 \%$ & $75 \%$ & $75 \%$ \\
\hline experiment 2 & sham control & & & & & & & & & & \\
\hline omissions (number of trials per session) & $0.2(0.0)$ & $0.9(0.2)$ & $0.0(0.0)$ & $0.0(0.0)$ & $0.0(0.0)$ & $0.0(0.0)$ & & $0.2(0.2)$ & $0.0(0.0)$ & $0.0(0.0)$ & $0.2(0.2)$ \\
\hline \multirow[t]{2}{*}{ response latency (s) } & $0.9(0.1)$ & $1.3(0.2)$ & $0.9(0.2)$ & $0.8(0.1)$ & $0.8(0.1)$ & $0.9(0.1)$ & & $0.8(0.1)$ & $0.8(0.1)$ & $0.8(0.1)$ & $0.8(0.1)$ \\
\hline & AcbS DA depletion & & & & & & \# & & & & \\
\hline omissions (number of trials per session) & $0.3(0.1)$ & $1.2(0.5)$ & $0.0(0.0)$ & $0.1(0.1)$ & $0.1(0.1)$ & $0.0(0.0)$ & & $0.1(0.1)$ & $0.0(0.0)$ & $0.0(0.0)$ & $0.0(0.0)$ \\
\hline response latency $(\mathrm{s})$ & $1.1(0.1)$ & $2.0(0.2)$ & $1.1(0.1)$ & $0.8(0.1)$ & $0.8(0.1)$ & $0.9(0.1)$ & & $0.9(0.1)$ & $0.8(0.1)$ & $0.7(0.1)$ & $0.7(0.1)$ \\
\hline experiment 3 & sham control & & & & & & & & & & \\
\hline omissions (number of trials per session) & $0.3(0.2)$ & $0.6(0.3)$ & $0.2(0.1)$ & $0.4(0.2)$ & $0.4(0.2)$ & $0.1(0.1)$ & & $0.4(0.2)$ & $0.3(0.3)$ & $0.2(0.1)$ & $0.4(0.2)$ \\
\hline \multirow[t]{2}{*}{ response latency (s) } & $1.1(0.1)$ & $1.4(0.2)$ & $1.0(0.1)$ & $0.9(0.1)$ & $0.9(0.1)$ & $1.4(0.1)$ & & $1.0(0.1)$ & $0.9(0.1)$ & $0.9(0.1)$ & $0.8(0.1)$ \\
\hline & OFC DA depletion & & & & & & & & & & \\
\hline omissions (number of trials per session) & $0.2(0.1)$ & $0.4(0.3)$ & $0.0(0.0)$ & $0.1(0.1)$ & $0.0(0.0)$ & $0.2(0.1)$ & & $0.1(0.1)$ & $0.0(0.0)$ & $0.2(0.1)$ & $0.1(0.1)$ \\
\hline \multirow[t]{2}{*}{ response latency (s) } & $1.1(0.1)$ & $1.6(0.2)$ & $1.0(0.1)$ & $0.9(0.1)$ & $0.9(0.1)$ & $1.1(0.2)$ & & $1.2(0.2)$ & $1.0(0.1)$ & $0.9(0.1)$ & $0.8(0.1)$ \\
\hline & & \multicolumn{6}{|c|}{$\# \mathrm{p}<0.01$ probability $\mathrm{x}$ treatment interaction (ANOVA) } & & & & \\
\hline
\end{tabular}


Pre- and post-surgery, the preference for the large/risky lever became increasingly lower in animals of both treatment groups over subsequent days with declining odds to obtain the large/risky reward (Fig. 4a). An overall ANOVA with the factors treatment group (sham, lesion) and surgery (pre-surgery, post-surgery) revealed no main effects of treatment group $(\mathrm{F}(1,22)=0.23$, n.s. $)$ and surgery $(\mathrm{F}(1,22)=0.001$, n.s. $)$ and no surgery $\times$ treatment group interaction $(F(1,22)=$ 0.46, n.s). However, the ANOVA showed a main effect of days $(\mathrm{F}(4,88)=61.21, \mathrm{p}<0.001)$ and a surgery $\times$ day interaction $(\mathrm{F}(4,88)=3.94, \mathrm{p}<0.01)$ but no day $\times$ treatment interaction $(\mathrm{F}(4,88)=0.18$, n.s. $)$ and no surgery $\times$ day $\times$ treatment interaction $(\mathrm{F}(4,88)=0.26$, n.s. $)$. A separate ANOVA on post-surgery data confirmed that choice behavior of sham controls and animals with AcbS DA depletion was sensitive to changes of probabilities to obtain the large/risky reward. There was no main effect of treatment, $(F(1,22)=$ 0.47 , n.s.) but of days $(F(4,88)=28.61, \mathrm{p}<0.001)$, and no
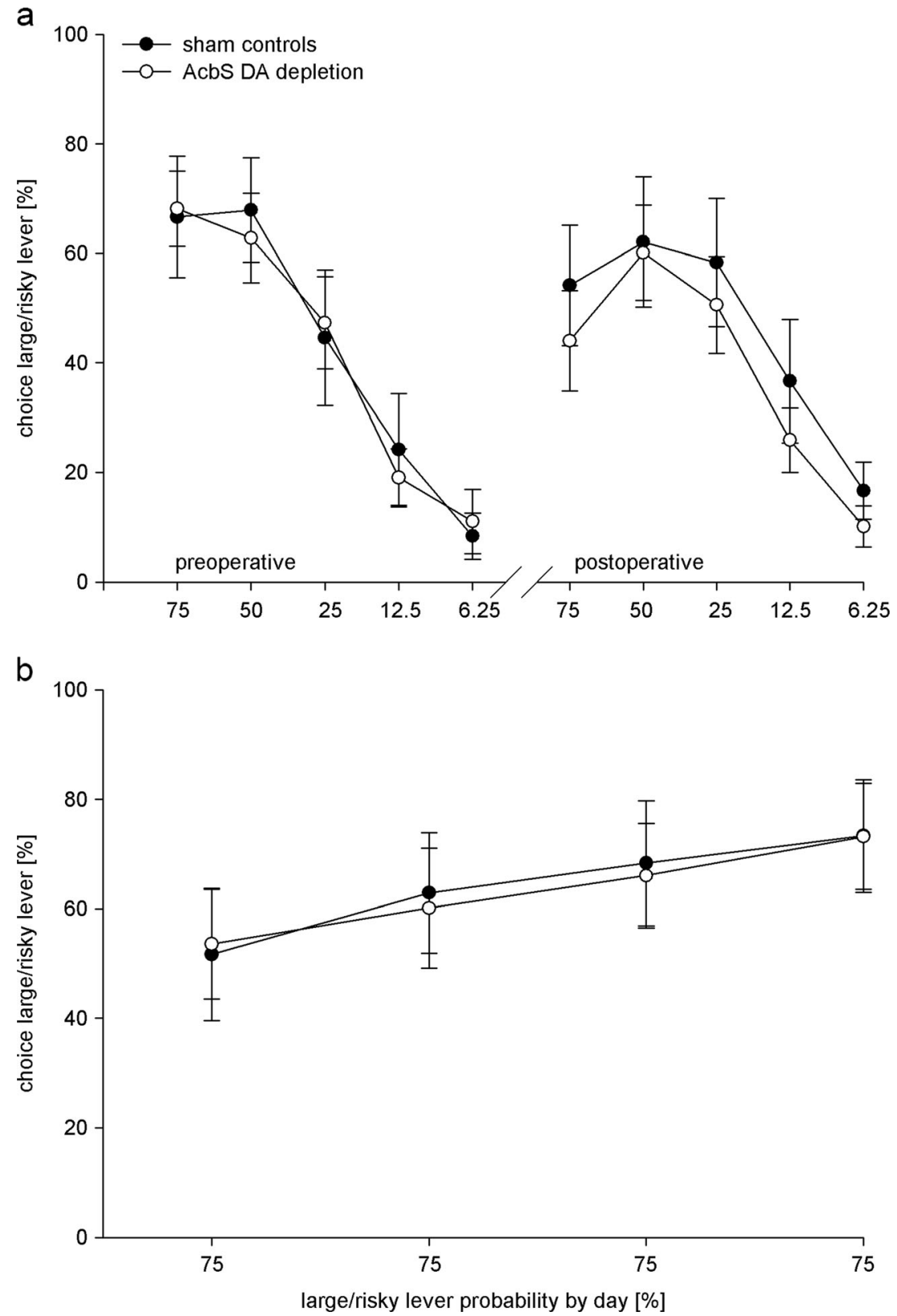

Fig. 4 Effects of 6-OHDA lesions of the AcbS on probabilistic choice. Mean $( \pm$ SEM) percentages of large/risky lever choices per day in shamlesioned ( $\mathrm{n}=10$, filled circles) and 6-OHDA-lesioned $(\mathrm{n}=14$, open circles) rats are given. (a) Percentage choice for the large/risky lever in the pDISC task is depicted as a function of the large/risky lever probability by day. During pre- and post-operative testing there were no differences between treatment groups. (b) Percentage choice for the large/ risky lever over days in the pFIX task is depicted. Treatment groups did not differ significantly 
day $\times$ treatment interaction $(F(4,88)=0.23$, n.s. $)$. Moreover, the number of trial omissions did not differ between sham controls and animals with AcbS DA depletion (Table 2). An ANOVA revealed no main effect of treatment $(F(1,22)=0.69$, n.s. $)$ but of days $(F(4,88)=11.10, p<0.001)$ and there was no day $\times$ treatment interaction $(F(4,88)=0.22$, n.s. $)$. In addition, relative to sham controls, animals with AcbS DA depletion had increased response latencies (Table 2). An ANOVA showed no main effects of treatment $(\mathrm{F}(1,22)=1.19$,n.s. $)$, but of days $(\mathrm{F}(4,88)=25.38, \mathrm{p}<0.001)$, and a day $\times$ treatment interaction $(\mathrm{F}(4,88)=4.96, \mathrm{p}<0.01)$.

Given the considerable pre- and post-surgical variability in choice behavior, we performed an additional analysis to assess the potential influence of individual risk preferences. Based on their preference for the large/risky lever in the initial preoperative session (four pellets at $p=0.75$ ), half of the rats in each treatment group were categorized as either risk-seeking or risk-avoiding using a median split based upon the respective group. An ANOVA with the factors risk preference (risk-seeking, risk-averse), treatment group (sham, lesion), and surgery (pre-surgery, post-surgery) revealed a main effects of risk preference $(F(1,20)=5.2, p<$ 0.05 ) but no risk preference $\times$ surgery $\times$ treatment group interaction $(\mathrm{F}(1,20)=3.2$, n.s). Thus, there is no evidence that AcbS DA depletion selectively impaired risky choice, for instance, in animals classified as being more risky during pre-surgical training.
As animals with AcbS DA depletion displayed a moderately reduced preference for the large/risky lever at $p=0.75$, we subsequently used the pFIX-task to test for their sensitivity to assess constant odds over four consecutive days (Fig. 4b). Preferences for the large/risky lever were similar in both treatment groups but increased moderately over days, an observation that possibly reflects learning. Accordingly, an ANOVA revealed no main effect of treatment $(F(1,22)=0.003$, n.s. $)$ but a significant main effect of days $(F(3,66)=13.20, \mathrm{p}<$ $0.001)$ and no day $\times$ treatment interaction $(\mathrm{F}(3,66)=0.20$, n.s). Furthermore, the number of trial omissions did not differ between sham controls and animals with AcbS DA depletion (Table 2). An ANOVA revealed no main effect of treatment $(\mathrm{F}(1,22)=1.89$, n.s. $)$ and of days $(\mathrm{F}(3,66)=1.18$, n.s. $)$ and there was no day $\times$ treatment interaction $(F(3,66)=0.60$, n.s.). In addition, response latencies did not differ between sham controls and animals with AcbS DA depletion (Table 2). An ANOVA showed no main effects of treatment $(F(1,22)=$ 0.001 ,n.s. $)$, and of days $(F(3,66)=2.66$, n.s. $)$ and no day $\times$ treatment interaction $(\mathrm{F}(3,66)=1.69$, n.s.).

In a separate group of otherwise intact rats trained on the pFIX task, we assessed the effects of systemic flupenthixol $(0.25 \mathrm{mg} / \mathrm{kg}$, i.p.) on a single test day (Fig. 5). Results revealed that, relative to vehicle controls, animals that received flupenthixol displayed a significantly reduced preference for the large/risky lever (vehicle, $97.6 \pm 1.8 \%$; flupenthixol, 86.6 $\pm 3.4 \%$; t-test: $\mathrm{t}(11)=2.99, \mathrm{p}=0.012)$. Moreover, relative to no treatment

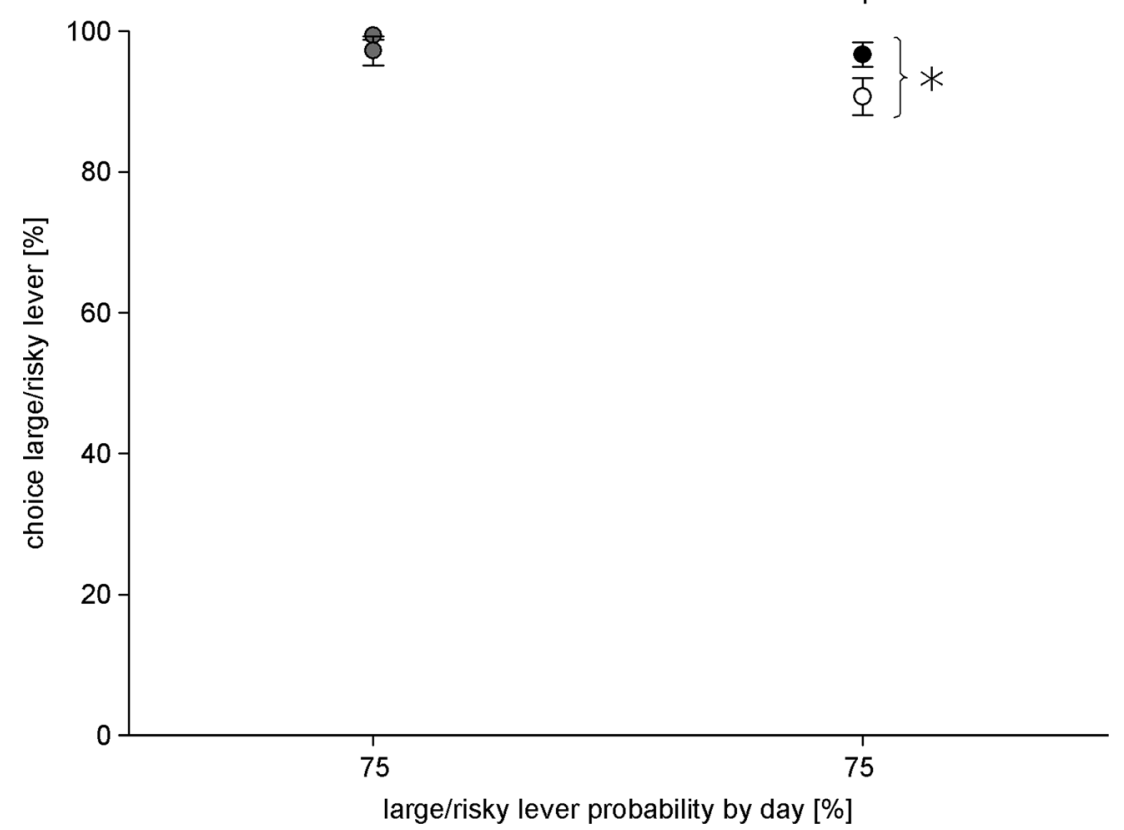

Fig. 5 Mean percentages $( \pm$ SEM) of large/risky lever choices in the pFIX task on the last training day ("no treatment") and on the test day, i.e., after systemic administration of flupenthixol $(0.25 \mathrm{mg} / \mathrm{kg}$, intraperitoneally (i.p.); $\mathrm{n}=6)$ and vehicle ( $1 \mathrm{ml} / \mathrm{kg}$, i.p., $\mathrm{n}=7$ ) ("treatment"), are depicted. *p $<0.05$, t-test 


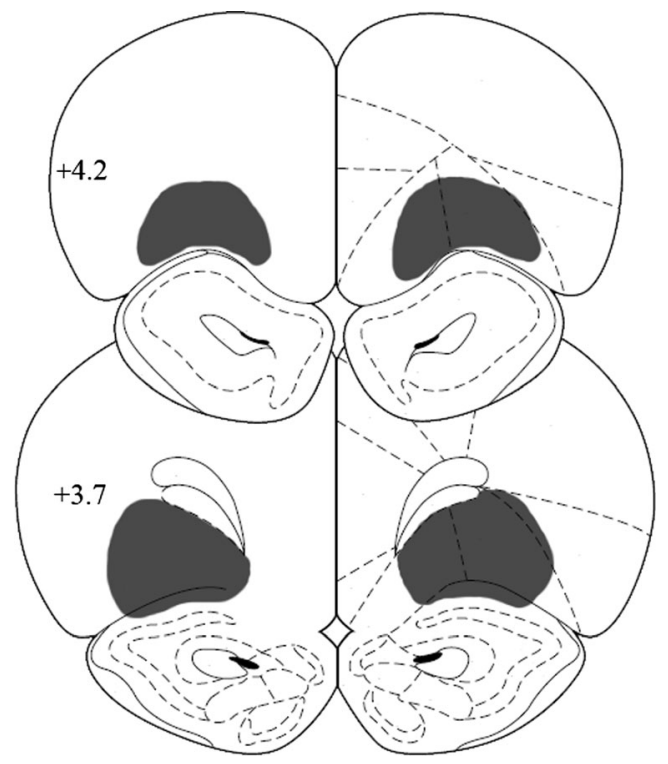

Fig. 6 Loss of TH-positive fibers in the OFC of 6-OHDA-lesioned rats. Qualitative reconstruction of the prefrontal region with, relative to sham controls, reduced TH-staining. The numbers indicate the distances from bregma, in millimeters

vehicle controls, animals that received flupenthixol showed similar response latencies (vehicle, $0.8 \pm 0.1 \mathrm{~s}$; flupenthixol, $1.3 \pm 0.4$ s; t-test: $\mathrm{t}(11)=1.37$, n.s. $)$ and an increased number of trial omissions (vehicle, $0.0 \pm 0.0$; flupenthixol, $13.7 \pm 8.2$; t-test: $\mathrm{t}(11)=1.81, \mathrm{p}=0.098)$.

\section{Experiment 3}

Figure 6 indicates damaged areas on stereotaxic atlas templates from Paxinos and Watson (1998). No animal was excluded due to misplaced OFC DA depletions. TH-positive fibers were present in the OFC of sham-lesioned rats, but rare in rats with 6-OHDA lesions. The areas that were nearly devoid of TH immunoreactivity appeared from about $4.2 \mathrm{~mm}$ to $3.7 \mathrm{~mm}$ relative to bregma and were restricted to the lateral and ventrolateral OFC. One animal was excluded due to strong bias to the small/certain lever throughout all preoperative days regardless of the actual odds of receiving large reward ( $>90 \%$ ), and three animals died during or after surgery. Final sample sizes were as follows: sham controls, $\mathrm{n}=$ 10 , rats with OFC DA depletion, $\mathrm{n}=16$.

Pre- and post-surgery, the preference for the large/risky lever became increasingly lower in animals of both treatment groups over subsequent days with declining odds to obtain the large/risky reward (Fig. 7a). An overall ANOVA with the factors treatment group (sham, lesion) and surgery (pre-surgery, post-surgery) showed no main effects of treatment $(F(1,24)=$ 0.48 , n.s. $)$ and surgery $(F(1,24)=0.33$, n.s. $)$ and no surgery $\times$ treatment interaction $(\mathrm{F}(1,24)=0.42, \mathrm{n} . \mathrm{s})$. In addition, the ANOVA showed a main effect of days $(\mathrm{F}(4,96)=67.06, \mathrm{p}$ $<0.001)$ but no surgery $\times$ day interaction $(\mathrm{F}(4,96)=1.02$, n.s.), no day $\times$ treatment interaction $(F(4,96)=0.25$, n.s. $)$ and no surgery $\times$ day $\times$ treatment interaction $(F(4,96)=0.55$, n.s. $)$. A separate ANOVA on post-surgery data confirmed that animals with sham lesion and OFC DA depletions were sensitive to changing odds of obtaining the large reward across days, i.e., there was no main effect of treatment $(F(1,24)=0.77$, n.s.) but of days $(F(4,96)=31.46, p<0.001)$, and there was no day $\times$ treatment interaction $(F(4,96)=0.38, n . s)$. Relative to sham controls, the number of trial omissions was moderately reduced in animals with OFC DA depletion (Table 2). An ANOVA revealed trends for treatment $(\mathrm{F}(1,24)=3.24, \mathrm{p}=$ $0.08)$ and day effects $(\mathrm{F}(4,96)=2.17, \mathrm{p}=0.08)$ and no day $\times$ treatment interaction $(\mathrm{F}(4,96)=0.64$, n.s.). Furthermore, response latencies in both treatment groups did not differ (Table 2). An ANOVA revealed no main effects of treatment $(\mathrm{F}(1,24)=0.02$, n.s. $)$ but of days $(\mathrm{F}(4,96)=16.99, \mathrm{p}<0.001)$, and no day $\times$ treatment interaction $(\mathrm{F}(4,96)=1.55$, n.s.). As described in Experiment 2, rats were categorized as either riskseeking or risk-avoiding using a median split. An ANOVA with the factors risk preference (risk-seeking, risk-averse), treatment group (sham, lesion), and surgery (pre-surgery, post-surgery) revealed a main effect of risk preference $(F(1$, $20)=4.72, p<0.05)$ but no risk preference $\times$ surgery $\times$ treatment group interaction $(\mathrm{F}(1,20)=0.08, \mathrm{n} . \mathrm{s})$. Thus, there is no evidence that OFC DA depletion selectively impaired risky choice, for example in animals classified as being more risky during pre-surgical training.

We investigated animals subsequently in the pFIX-task to test for their sensitivity to assess constant odds over four consecutive days (Fig 7b). Preferences for the large/risky lever were similar in both treatment groups but increased moderately over days, possibly reflecting learning. Accordingly, An ANOVA revealed no main effect of treatment $(\mathrm{F}(1,24)=$ 0.57 , n.s. $)$ but of days $(\mathrm{F}(3,72)=39.67, \mathrm{p}<0.001)$, and a trend for a day $\times$ treatment interaction $(\mathrm{F}(3,72)=2.33$, $\mathrm{p}=$ 0.08). Furthermore, the number of trial omissions did not differ between sham controls and animals with OFC DA depletion (Table 2). An ANOVA revealed no main effect of treatment $(F(1,24)=2.44$, n.s. $)$ and of days $(F(3,72)=0.33$, n.s. $)$ and no day $\times$ treatment interaction $(\mathrm{F}(3,72)=0.77$, n.s. $)$. In addition, response latencies were similar in sham controls and animals with OFC DA depletion (Table 2). An ANOVA showed no main effects of treatment $(\mathrm{F}(1,24)=0.37$,n.s. $)$, but of days $(\mathrm{F}(3,72)=5.45, \mathrm{p}<0.05)$ and no day $\times$ treatment interaction $(\mathrm{F}(3,72)=1.18$, n.s. $)$.

\section{Discussion}

Here we show that the capacity to assess the magnitude and likelihood of rewards associated with alternative courses of action does not rely on DA input to the AcbS or OFC. 

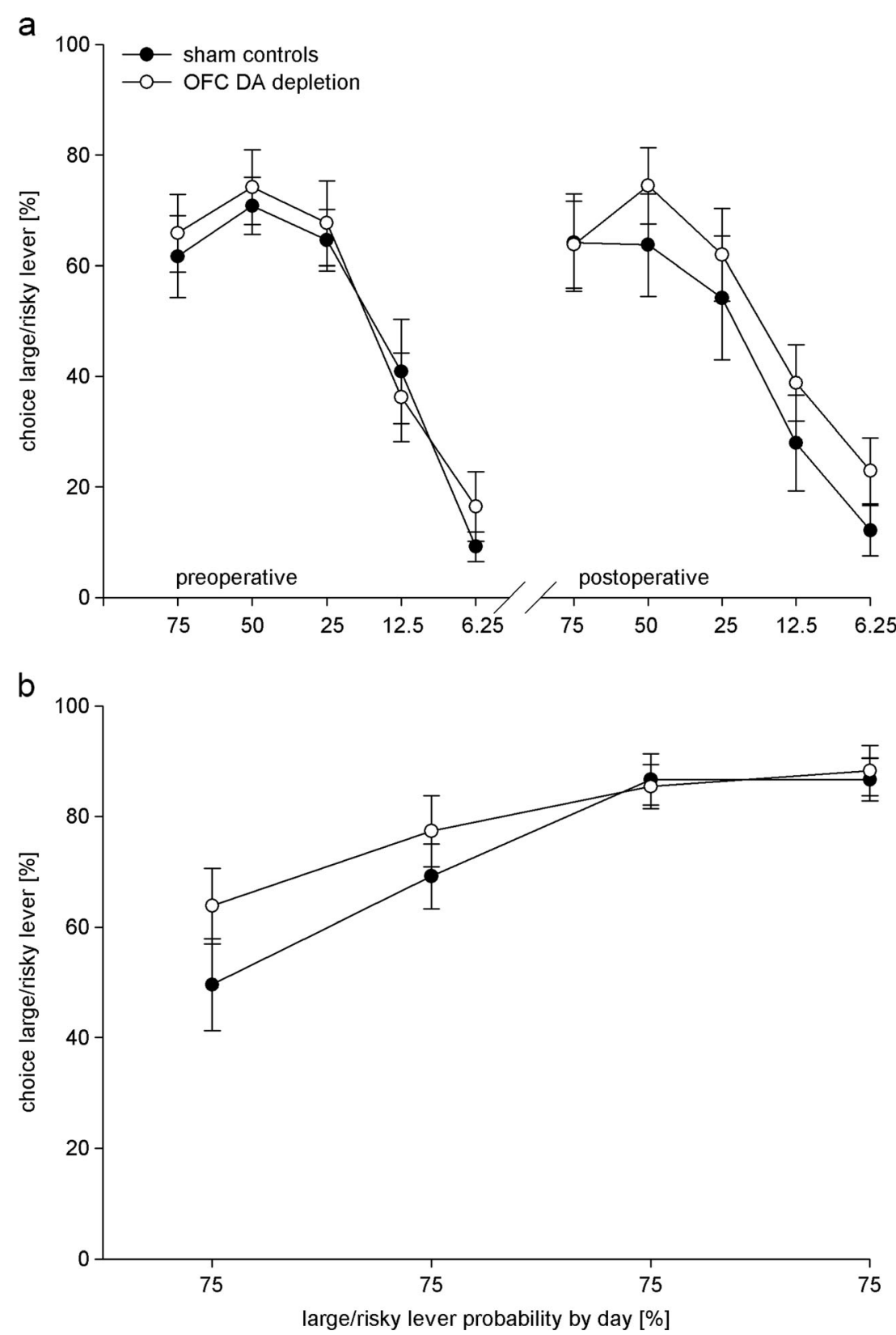

Fig. 7 Effects of 6-OHDA lesions of the OFC on probabilistic choice. Mean percentages $( \pm$ SEM) of large/risky lever choices per day in shamlesioned ( $\mathrm{n}=10$, filled circles) and 6-OHDA-lesioned $(\mathrm{n}=16$, open circles) rats are given. (a) Percentage choice for the large/risky lever in the pDISC task is depicted as a function of the large/risky lever

\section{Probabilistic choice task}

In this study we used a probabilistic choice task in which rats chose between two levers, a small/certain lever that delivered one food pellet at $\mathrm{p}=1$ and a large/risky lever that delivered four food pellets with decreasing probabilities across fivve subsequent test days $(\mathrm{p}=0.75,0.5,0.25,0.125,0.0625$, respectively) with one session per day. In all experiments, control animals displayed probabilistic discounting over days, and, consistent with predictions of the value matching law probability by day. During pre- and post-operative testing there were no differences between treatment groups. (b) Percentage choice for the large/ risky lever over days in the pFIX task is depicted. Treatment groups did not differ significantly

(Herrnstein, 1961), showed indifferent choice behavior if the large/risky and small/certain lever had about the same expected value (i.e., four pellets at $\mathrm{p}=0.25$ vs. one pellet at $\mathrm{p}=1$ ). Moreover, in line with theoretical (Herrnstein, 1961) and empirical (Kacelnik \& Bateson, 1997) accounts, control animals in our experiments exhibited considerable risk aversion if the large/risky lever had a low expected value compared to the small/certain lever (i.e., four pellets at $p=0.0625 \mathrm{vs}$. one pellet at $\mathrm{p}=1)$. Overall, these data are consistent with earlier findings using a very similar task (Nasrallah, et al., 2009) and 
suggest that this task may be sensitive to experimental manipulations of DA transmission. In line with this notion, amphetamine at a dose previously shown to alter choice behavior in various other instrumental tasks (Evenden \& Robbins, 1983a, 1983b) increased the preference for the large/risky lever. Likewise, a large body of evidence suggests that amphetamine can increase or decrease risk-taking (Mitchell, Vokes, Blankenship, Simon, \& Setlow, 2011; St Onge \& Floresco, 2009) depending on the type of task (Cocker, Dinelle, Kornelson, Sossi, \& Winstanley, 2012; St Onge, Chiu, \& Floresco, 2010), training protocol (St Onge, et al., 2010) or individual preferences for risk-taking (Cocker, et al., 2012). In addition, results reveal that cocaine at a dose that impaired rats' sensitivity to changes in punishment risk (Simon, et al., 2009) tended to increase in our rats the preference for the large/risky lever on the first 3 days, yet the day $\times$ treatment interaction nearly missed significance $(p=0.06)$. The lack of effects on the last two test days with lower large reward probabilities might not be accounted for by behavioral tolerance as such an effect was not observed after repeated injections of cocaine of the same dose as used here $(3 \times 15 \mathrm{mg} / \mathrm{kg} ; 1 \mathrm{~h}$ apart $)$ (Caster, Walker, \& Kuhn, 2005). Correspondingly, tolerance to the effects of psychostimulants on cognition has only been demonstrated with considerably higher doses and much longer regimens (Dalley et al., 2005; Simon, Mendez, \& Setlow, 2007). Furthermore, repeated administration of amphetamine and cocaine can induce behavioral sensitization (Pierce and Kalivas, 1997). In amphetaminetreated, but not cocaine-treated animals, the number of trial omissions became increasingly higher over days. We can not exclude that this observation reflects to some extent behavioral sensitization to the effects of amphetamine, e.g., stereotypies competing with lever pressing.

Overall, the task used here was sensitive to changes of risktaking induced by two prototypical DAergic drugs that have been previously shown to alter risk-based decision-making in rats (Simon, et al., 2009; St Onge \& Floresco, 2009) and humans (Rogers, 2011; Verdejo-Garcia et al., 2007). However, mechansims through which amphetamine or cocaine could increase risk-taking in our task are likely to be complex and not necessarily identical given that these drugs differ in part in their monoamine transporter affinities and can both increase or decrease risk-taking in a dose- and taskdependent manner (see Simon et al. 2009, for a comprehensive discussion). Of note, the risky decision-making task used in our study employed reward omission probabilities associated with the large reward as the discounting factor. Thus, it is conceivable that stimulation of mesolimbic DA transmission by amphetamine and cocaine amplifies the influence of rewarding stimuli thereby shifting choice towards the large, risky reward.

\section{AcbS dopamine and probabilistic choice}

Unlike in sham controls, TH-positive fibers in the AcbS were rare in lesioned rats. These findings are consistent with previous data demonstrating that intra-AcbS infusions of the same or lower doses of 6-OHDA clearly reduced the striatal DA innervation assessed by an analysis of DA transporter density (Boye, Grant, \& Clarke, 2001) and DA tissue content (Nelson, Thur, Horsley, Spicer, Marsden, \& Cassaday 2011; Sokolowski \& Salamone, 1998). Likewise, intraAcbC infusions of 6-OHDA at a lower dose markedly decreased TH-immunoreactivity (Lex \& Hauber, 2010b; Mai \& Hauber, 2012; Mai, Sommer, \& Hauber, 2012). It should be noted that in most lesioned rats, THimmunoreactivity was also reduced in an area restricted to the most medial part of adjacent AcbC (Fig. 3). As 6-OHDA-induced loss of striatal DA terminals remained stable over 4 weeks (Blandini, Levandis, Bazzini, Nappi, \& Armentero, 2007), it is unlikely that DA depletion recovered during the course of our experiments.

Behavioral results demonstrate that, like sham controls, rats with AcbS DA depletion were sensitive to changes of probabilities to obtain the large/risky reward across days but displayed increased response latencies. Of note, post-surgery, sham controls do not show a discernible bias to the large/risky reward lever during high probability blocks, but a marked risk-aversion during low probability blocks. By contrast, in other studies (St Onge \& Floresco, 2009; Stopper \& Floresco, 2011) sham controls displayed a considerable bias to the large/ risky reward lever during high probability blocks but relatively little risk-aversion during low probability blocks. Together these findings suggest that rats develop a bias towards the more advantageous option either during the high or during the low large reward probability blocks.

Given the considerable pre- and post-surgical variability in choice behavior, particularly at higher probability blocks, we performed an additional analysis to assess the potential influence of individual risk preferences. Results suggest that, using the preference for the large/risky lever in the pre-operative session with indifferent choice as criterion, rats exhibited individual differences in risk-taking behavior, i.e., there were subgroups of rats that were more risk-seeking or risk-averse. While such individual differences in risk preferences might in part explain enhanced variability of pre- and post-surgical choice, we found no evidence that, for instance, AcbS DA depletion preferentially altered choice in animals considered as risk-seeking. The preference for the large reward/risky lever in particular at $p=0.75$ seemed to be marginally reduced in lesioned rats. Therefore, we subsequently used a task variant to test for their sensitivity to assess large reward at constant high odds $(\mathrm{p}=0.75)$ versus small/certain reward 
over four consecutive days. However, we found no evidence for a shift in choice behavior in rats with AcbS DA depletions relative to sham controls. Previous studies suggest that the Acb as such supports risky choice in tasks with fixed reward probabilities when the risky option was more advantageous in the long term (Stopper and Floresco, 2011); however, in view of considerable methodological discrepancies between these studies, respective conclusions are difficult to draw.

As yet, little is known about the role of the AcbS and its DA innervation in risk-based decision-making. The available evidence suggest that, unlike AcbC lesions, AcbS lesions had no effects on choice between delayed, certain and immediate, uncertain rewards (Pothuizen, Jongen-Relo, Feldon, \& Yee, 2005). By contrast, in a probabilistic learning and reversal task, AcbS inactivation reduced selection of those actions that more likely yield reward (Dalton, Phillips, \& Floresco, 2014). Furthermore, in a risk discounting task that involved withinsession shifts of large reward probabilities, inactivation of the AcbS reduced the bias toward larger-magnitude rewards (Stopper \& Floresco, 2011), suggesting that the AcbS may play a subtle and task-dependent role in risk-based decisionmaking. DA signals in the whole Acb support risk-based decision-making (Stopper, et al., 2013); however, the selective contribution of DA signals in the AcbS has not been investigated so far. Our data suggest that AcbS DA input may not play a major role to support the capacity to evaluate magnitude and likelihood of rewards associated with different levers, at least in a probabilistic choice task that involves probability discounting across sessions with fixed within-session reward probabilities. However, it is possible that AcbC DA depletion, similar to intra-Acb infusion of DA receptor antagonists (Stopper, et al., 2013), alters risky choice in tasks that involve within-session shifts of reward probabilities (Cardinal \& Howes, 2005; St Onge \& Floresco, 2009) demanding more complex cognitive processes, i.e., a persistent evaluation and updating of the current values associated with the available response options.

\section{OFC dopamine and probabilistic choice}

Our histologic analysis demonstrates in lesioned rats a loss of TH-positive fibers largely limited to the OFC. Correspondingly, the same dose of 6-OHDA, if infused into the adjacent medial prefrontal cortex, markedly reduced the DA innervation around the infusion site (Lex \& Hauber, 2010a; Mai \& Hauber, 2012; Pycock, Carter, \& Kerwin, 1980). As DA depletions after prefrontal 6-OHDA injections are persistent (Bubser \& Schmidt, 1990), recovery of DA terminals seems unlikely. Thus, the failure to detect behavioral changes in our lesioned animals may not reflect behavioral plasticity mediated by residual OFC DA. Consistent with this notion, cortical DA depletion using a similar protocol produced persistent impairments of other forms of cost/ benefit-related decision-making (Nelson, Cooper, Thur, Marsden, \& Cassaday, 2011; Schweimer, Saft, \& Hauber, 2005). It is well known that mPFC 6-OHDA injections can also reduce noradrenaline (NA) levels, although to a lesser extent than DA levels, even after pretreatment with the NA/ serotonin reuptake inhibitor desipramine (which we did not use) (e.g., Koch and Bubser, 1994; Morrow, Elsworth, Rasmusson and Roth, 1999. Thus, we cannot exclude the possibility that the NA input into the OFC was affected in our rats as well. To our knowledge, the role of NA in riskbased decision-making is unknown to date.

Like sham controls, rats with OFC DA depletion were sensitive to changes of probabilities to obtain the large/risky reward across days. In addition, if tested in a task variant, lesioned rats, like sham controls, displayed a preference for constant odds $(p=0.75)$ for large reward over small/certain reward across four test days. Considerable evidence implicated the OFC in risk-taking; however, its exact role is still a matter of debate. For instance, transient or permanent OFC inactivation increased, decreased, or left unaffected risktaking and these mixed effects might be accounted for in part by the type of task, time point of inactivation during task acquisition, or targeted OFC subregion (Floresco, St Onge, Ghods-Sharifi, \& Winstanley, 2008; Ishii, Ohara, Tobler, Tsutsui, \& Iijima, 2012; Jentsch, Woods, Groman, \& Seu, 2010; Mobini et al., 2002; Pais-Vieira, Lima, \& Galhardo, 2007; St Onge \& Floresco, 2010; Zeeb \& Winstanley, 2011). Of note, a recent study using a probabilistic discounting task similar to the one used here showed that OFC lesions promoted risk aversion (Abela \& Chudasama, 2013). This latter and our present findings imply that the capacity to assess the magnitude and likelihood of rewards in such tasks does rely on the integrity of the OFC but appears not to be not supported by OFC DA input. However, given that inactivation of the medial but not lateral OFC altered risky choice in well-trained rats tested in a probabilistic discounting task (St. Onge and Floresco, 2010; Stopper, Green, \& Floresco, 2014), the possibility remains that OFC DA depletions in our study that were induced by three 6OHDA microinfusions per hemisphere at different anterioposterior/mediolateral coordinates did only partially target the medial OFC. A preliminary analysis (data not shown) revealed that a subgroup of rats in which reduced $\mathrm{TH}$ immunoreactivity was most evident in the anteromedial OFC showed a trend for increased risk-taking relative to the remaining rats with OFC DA depletion. However, this analysis is limited as reduced $\mathrm{TH}$ staining in these lesion subgroups also varies in targeted more caudal OFC regions. Additional research using focal medial vs. lateral OFC DA depletions will be needed to address this issue. 


\section{Concluding comments}

In a probabilistic choice task that involved within-session shifts of reward probabilities, a blockade or stimulation of DA signaling in the MPFC or Acb altered different components of risk-based decision-making (Nelson, Cooper, et al., 2011; St Onge, et al., 2011; Stopper, et al., 2013). By contrast, in less demanding probabilistic choice tasks that involved fixed within-session probabilities that were shifted across subsequent sessions, risk-based decision-making including probabilistic discounting was intact after mPFC and AcbC DA depletion (Mai \& Hauber, 2012) and, as shown here, OFC and AcbS DA depletion. Importantly, our present study also shows that amphetamine or cocaine-induced enhancement of DA activity induced profound changes in a probabilistic choice task that involved fixed within-session probabilities. Moreover, ethanol exposure produced abnormal AcbC DA signaling to risk-impaired choice behavior in a similar task (Nasrallah, et al., 2011). Together, these findings point to the view that, in tasks such as used here, the capacity to evaluate the magnitude and likelihood of rewards associated with alternative courses of action is sensitive to aberrant or increased DA signaling but insensitive to DA depletion in the mPFC, OFC, and Acb.

Of note, patients with Parkinson' disease (PD), a disorder associated with midbrain DA depletion and associated areas, show deficits in risk-based decision-making; however, data are mixed in part due to the influence of DA medications and the use of different behavioral tasks (Clark \& Dagher, 2014; Santangelo, Barone, Trojano, \& Vitale, 2013; Sharp et al., 2013). Nevertheless, one of the few available studies that investigated decisions under risk in unmedicated PD patients revealed that they were similar to controls when evaluating magnitude and probability of gains, but more sensitive to losses relative to controls (Sharp, et al., 2013). Furthermore, the first neuroimaging study that assessed risky decisionmaking in a two-choice gambling task revealed intact choice behavior in newly diagnosed drug-naïve PD patients but reduced neural responses to the reward outcome in many core regions of the meso-corticolimbic DA system including the ventral tegmental area, ventral striatum, or orbitofrontal cortex (van der Vegt et al., 2013). In other words, many regions of the meso-corticolimbic DA system are already compromised in the early stages of unmedicated PD without concomitant changes in risk-based decision-making. These clinical findings bear similarities to our present and previous (Mai and Hauber, 2012) rodent data suggesting that a loss of DA activity in the mPFC, OFC, AcbC, or AcbS per se is not sufficient to alter probabilistic choice in simple tasks that require an evaluation of the probability of fixed within-session gains.

Acknowledgments This study was supported by a grant from the Deutsche Forschungsgemeinschaft (HA2340/9-1).

\section{References}

Abela, A. R., \& Chudasama, Y. (2013). Dissociable contributions of the ventral hippocampus and orbitofrontal cortex to decision-making with a delayed or uncertain outcome. The European Journal of Neuroscience, 37(4), 640-647. doi:10.1111/ejn.12071

Blandini, F., Levandis, G., Bazzini, E., Nappi, G., \& Armentero, M. T. (2007). Time-course of nigrostriatal damage, basal ganglia metabolic changes and behavioural alterations following intrastriatal injection of 6-hydroxydopamine in the rat: new clues from an old model. The European Journal of Neuroscience, 25(2), 397-405. doi:10. 1111/j.1460-9568.2006.05285.x

Boye, S. M., Grant, R. J., \& Clarke, P. B. (2001). Disruption of dopaminergic neurotransmission in nucleus accumbens core inhibits the locomotor stimulant effects of nicotine and D-amphetamine in rats. Neuropharmacology, 40(6), 792-805.

Bubser, M., \& Schmidt, W. J. (1990). 6-Hydroxydopamine lesion of the rat prefrontal cortex increases locomotor activity, impairs acquisition of delayed alternation tasks, but does not affect uninterrupted tasks in the radial maze. Behavioural Brain Research, 37(2), 157-168.

Cardinal, R. N., \& Howes, N. J. (2005). Effects of lesions of the nucleus accumbens core on choice between small certain rewards and large uncertain rewards in rats. BMC Neuroscience, 6, 37. doi:10.1186/ 1471-2202-6-37

Caster, J. M., Walker, Q. D., \& Kuhn, C. M. (2005). Enhanced behavioral response to repeated-dose cocaine in adolescent rats. Psychopharmacology, 183(2), 218-225. doi:10.1007/s00213-0050159-4

Clark, C. A., \& Dagher, A. (2014). The role of dopamine in risk taking: a specific look at Parkinson's disease and gambling. Frontiers in Behavioral Neuroscience, 8, 196. doi:10.3389/fnbeh.2014.00196

Cocker, P. J., Dinelle, K., Kornelson, R., Sossi, V., \& Winstanley, C. A. (2012). Irrational choice under uncertainty correlates with lower striatal $\mathrm{D}(2 / 3)$ receptor binding in rats. The Journal of Neuroscience, 32(44), 15450-15457. doi:10.1523/JNEUROSCI. 0626-12.2012

Dalley, J. W., Laane, K., Pena, Y., Theobald, D. E., Everitt, B. J., \& Robbins, T. W. (2005). Attentional and motivational deficits in rats withdrawn from intravenous self-administration of cocaine or heroin. Psychopharmacology, 182(4), 579-587. doi:10.1007/s00213005-0107-3

Dalton, G. L., Phillips, A. G., \& Floresco, S. B. (2014). Preferential involvement by nucleus accumbens shell in mediating probabilistic learning and reversal shifts. The Journal of Neuroscience, 34(13), 4618-4626. doi:10.1523/JNEUROSCI. 5058-13.2014

Evenden, J. L., \& Robbins, T. W. (1983a). Dissociable effects of d-amphetamine, chlordiazepoxide and alpha-flupenthixol on choice and rate measures of reinforcement in the rat. Psychopharmacology, 79(2-3), 180-186.

Evenden, J. L., \& Robbins, T. W. (1983b). Increased response switching, perseveration and perseverative switching following $\mathrm{d}$ amphetamine in the rat. Psychopharmacology, 80(1), 67-73.

Fiorillo, C. D., Tobler, P. N., \& Schultz, W. (2003). Discrete coding of reward probability and uncertainty by dopamine neurons. Science, 299(5614), 1898-1902.

Floresco, S. B., St Onge, J. R., Ghods-Sharifi, S., \& Winstanley, C. A. (2008). Cortico-limbic-striatal circuits subserving different forms of cost-benefit decision making. Cognitive, Affective and Behavioral Neuroscience, 8(4), 375-389. doi:10.3758/CABN.8.4.375

Herrnstein, R. J. (1961). Relative and absolute strength of response as a function of frequency of reinforcement. Journal of the Experimental Analysis of Behavior, 4, 267-272. doi:10.1901/jeab. 1961.4-267

Ishii, H., Ohara, S., Tobler, P. N., Tsutsui, K., \& Iijima, T. (2012). Inactivating anterior insular cortex reduces risk taking. The 
Journal of Neuroscience, 32(45), 16031-16039. doi:10.1523/ JNEUROSCI. 2278-12.2012

Jentsch, J. D., Woods, J. A., Groman, S. M., \& Seu, E. (2010). Behavioral characteristics and neural mechanisms mediating performance in a rodent version of the Balloon Analog Risk Task. Neuropsychopharmacology, 35(8), 1797-1806. doi:10.1038/npp. 2010.47

Kacelnik, A., \& Bateson, M. (1997). Risk-sensitivity: crossroads for theories of decision-making. Trends in Cognitive Sciences, 1(8), 304309.

Koch, M., \& Bubser, M. (1994). Deficient sensorimotor gating after 6hydroxydopamine lesion of the rat medial prefrontal cortex is reversed by haloperidol. The European Journal of Neuroscience, 6(12), 1837-1845.

Lex, B., \& Hauber, W. (2010a). The role of dopamine in the prelimbic cortex and the dorsomedial striatum in instrumental conditioning. Cerebral Cortex, 20(4), 873-883. doi:10.1093/cercor/bhp151

Lex, B., \& Hauber, W. (2010b). The role of nucleus accumbens dopamine in outcome encoding in instrumental and Pavlovian conditioning. Neurobiology of Learning and Memory, 93(2), 283-290. doi:10. 1016/j.nlm.2009.11.002

Mai, B., \& Hauber, W. (2012). Intact risk-based decision making in rats with prefrontal or accumbens dopamine depletion. Cognitive, Affective and Behavioral Neuroscience, 12(4), 719-729. doi:10. 3758/s13415-012-0115-9

Mai, B., Sommer, S., \& Hauber, W. (2012). Motivational states influence effort-based decision making in rats: the role of dopamine in the nucleus accumbens. Cognitive, Affective and Behavioral Neuroscience, 12(1), 74-84. doi:10.3758/s13415-011-0068-4

Mitchell, M. R., Vokes, C. M., Blankenship, A. L., Simon, N. W., \& Setlow, B. (2011). Effects of acute administration of nicotine, amphetamine, diazepam, morphine, and ethanol on risky decisionmaking in rats. Psychopharmacology, 218(4), 703-712. doi:10. 1007/s00213-011-2363-8

Mobini, S., Body, S., Ho, M. Y., Bradshaw, C. M., Szabadi, E., Deakin, J. F., \& Anderson, I. M. (2002). Effects of lesions of the orbitofrontal cortex on sensitivity to delayed and probabilistic reinforcement. Psychopharmacology, 160(3), 290-298.

Morrow, B. A., Elsworth, J. D., Rasmusson, A. M., \& Roth, R. H. (1999). The role of mesoprefrontal dopamine neurons in the acquisition and expression of conditioned fear in the rat. Neuroscience, 92(2), 553564.

Nasrallah, N. A., Clark, J. J., Collins, A. L., Akers, C. A., Phillips, P. E., \& Bernstein, I. L. (2011). Risk preference following adolescent alcohol use is associated with corrupted encoding of costs but not rewards by mesolimbic dopamine. Proceedings of the National Academy of Sciences of the United States of America, 108(13), 5466-5471. doi: 10.1073/pnas.1017732108

Nasrallah, N. A., Yang, T. W., \& Bernstein, I. L. (2009). Long-term risk preference and suboptimal decision making following adolescent alcohol use. Proceedings of the National Academy of Sciences of the United States of America, 106(41), 17600-17604. doi:10.1073/ pnas.0906629106

Nelson, A. J., Cooper, M. T., Thur, K. E., Marsden, C. A., \& Cassaday, H. J. (2011). The effect of catecholaminergic depletion within the prelimbic and infralimbic medial prefrontal cortex on recognition memory for recency, location, and objects. Behavioral Neuroscience, 125(3), 396-403. doi:10.1037/a0023337

Nelson, A. J., Thur, K. E., Horsley, R. R., Spicer, C., Marsden, C. A., \& Cassaday, H. J. (2011). Reduced dopamine function within the medial shell of the nucleus accumbens enhances latent inhibition. Pharmacology, Biochemistry and Behavior, 98(1), 1-7. doi:10. 1016/j.pbb.2010.11.025

O'Neill, M., \& Schultz, W. (2010). Coding of reward risk by orbitofrontal neurons is mostly distinct from coding of reward value. Neuron, 68(4), 789-800. doi:10.1016/j.neuron.2010.09.031
Pais-Vieira, M., Lima, D., \& Galhardo, V. (2007). Orbitofrontal cortex lesions disrupt risk assessment in a novel serial decision-making task for rats. Neuroscience, 145(1), 225-231.

Paxinos, G., \& Watson, C. (1998). The rat brain in stereotaxic coordinates (4th ed.). San Diego: Academic Press.

Pierce, R. C., \& Kalivas, P. W. (1997). A circuitry model of the expression of behavioral sensitization to amphetamine-like psychostimulants. Brain Research, 25(2), 192-216.

Poncelet, M., Chermat, R., Soubrie, P., \& Simon, P. (1983). The progressive ratio schedule as a model for studying the psychomotor stimulant activity of drugs in the rat. Psychopharmacology, 80(2), 184189.

Pothuizen, H. H., Jongen-Relo, A. L., Feldon, J., \& Yee, B. K. (2005). Double dissociation of the effects of selective nucleus accumbens core and shell lesions on impulsive-choice behaviour and salience learning in rats. The European Journal of Neuroscience, 22(10), 2605-2616. doi:10.1111/j.1460-9568.2005.04388.x

Pycock, C. J., Carter, C. J., \& Kerwin, R. W. (1980). Effect of 6hydroxydopamine lesions of the medial prefrontal cortex on neurotransmitter systems in subcortical sites in the rat. Journal of Neurochemistry, 34(1), 91-99.

Rogers, R. D. (2011). The roles of dopamine and serotonin in decision making: evidence from pharmacological experiments in humans. Neuropsychopharmacology, 36(1), 114-132. doi:10.1038/npp. 2010.165

Roitman, J. D., \& Roitman, M. F. (2010). Risk-preference differentiates orbitofrontal cortex responses to freely chosen reward outcomes. The European Journal of Neuroscience, 31(8), 1492-1500. doi:10. 1111/j.1460-9568.2010.07169.x

Santangelo, G., Barone, P., Trojano, L., \& Vitale, C. (2013). Pathological gambling in Parkinson's disease. A comprehensive review. Parkinsonism and Related Disorders, 19(7), 645-653. doi:10. 1016/j.parkreldis.2013.02.007

Schweimer, J., Saft, S., \& Hauber, W. (2005). Involvement of catecholamine neurotransmission in the rat anterior cingulate in effort-related decision making. Behavioral Neuroscience, 119(6), 1687-1692. doi:10.1037/0735-7044.119.6.1687

Sharp, M. E., Viswanathan, J., McKeown, M. J., Appel-Cresswell, S., Stoessl, A. J., \& Barton, J. J. (2013). Decisions under risk in Parkinson's disease: preserved evaluation of probability and magnitude. Neuropsychologia, 51(13), 2679-2689. doi:10.1016/j. neuropsychologia.2013.08.008

Simon, N. W., Gilbert, R. J., Mayse, J. D., Bizon, J. L., \& Setlow, B. (2009). Balancing risk and reward: a rat model of risky decision making. Neuropsychopharmacology, 34(10), 2208-2217. doi:10. 1038/npp.2009.48

Simon, N. W., Mendez, I. A., \& Setlow, B. (2007). Cocaine exposure causes long-term increases in impulsive choice. Behavioral Neuroscience, 121(3), 543-549. doi:10.1037/0735-7044.121.3.543

Sokolowski, J. D., \& Salamone, J. D. (1998). The role of accumbens dopamine in lever pressing and response allocation: effects of 6OHDA injected into core and dorsomedial shell. Pharmacology, Biochemistry and Behavior, 59(3), 557-566.

St Onge, J. R., Abhari, H., \& Floresco, S. B. (2011). Dissociable contributions by prefrontal D1 and D2 receptors to risk-based decision making. The Journal of Neuroscience, 31(23), 8625-8633. doi:10. 1523/JNEUROSCI. 1020-11.2011

St Onge, J. R., Ahn, S., Phillips, A. G., \& Floresco, S. B. (2012). Dynamic fluctuations in dopamine efflux in the prefrontal cortex and nucleus accumbens during risk-based decision making. The Journal of Neuroscience, 32(47), 16880-16891. doi:10.1523/ JNEUROSCI. 3807-12.2012

St Onge, J. R., Chiu, Y. C., \& Floresco, S. B. (2010). Differential effects of dopaminergic manipulations on risky choice. Psychopharmacology, 211(2), 209-221. doi:10.1007/s00213-0101883-y 
St Onge, J. R., \& Floresco, S. B. (2009). Dopaminergic modulation of risk-based decision making. Neuropsychopharmacology, 34(3), 681-697.

St Onge, J. R., \& Floresco, S. B. (2010). Prefrontal cortical contribution to risk-based decision making. Cerebral Cortex, 20(8), 1816-1828. doi:10.1093/cercor/bhp250

Stopper, C. M., \& Floresco, S. B. (2011). Contributions of the nucleus accumbens and its subregions to different aspects of risk-based decision making. Cognitive, Affective and Behavioral Neuroscience, 11(1), 97-112. doi:10.3758/s13415-010-0015-9

Stopper, C. M., Khayambashi, S., \& Floresco, S. B. (2013). Receptorspecific modulation of risk-based decision making by nucleus accumbens dopamine. Neuropsychopharmacology, 38(5), 715-728. doi:10.1038/npp.2012.240

Stopper, C. M., Green, E. B., \& Floresco, S. B. (2014). Selective involvement by the medial orbitofrontal cortex in biasing risky, but not impulsive, choice. Cerebral Cortex, 24(1), 154-162. doi:10.1093/ cercor/bhs 297
Sugam, J. A., Day, J. J., Wightman, R. M., \& Carelli, R. M. (2012). Phasic nucleus accumbens dopamine encodes risk-based decisionmaking behavior. Biological Psychiatry, 71(3), 199-205. doi:10. 1016/j.biopsych.2011.09.029

van der Vegt, J. P., Hulme, O. J., Zittel, S., Madsen, K. H., Weiss, M. M., Buhmann, C., \& Siebner, H. R. (2013). Attenuated neural response to gamble outcomes in drug-naive patients with Parkinson's disease. Brain, 136(Pt 4), 1192-1203. doi:10.1093/brain/awt027

Verdejo-Garcia, A., Benbrook, A., Funderburk, F., David, P., Cadet, J. L., $\&$ Bolla, K. I. (2007). The differential relationship between cocaine use and marijuana use on decision-making performance over repeat testing with the Iowa Gambling Task. Drug and Alcohol Dependence, 90(1), 2-11. doi:10.1016/j.drugalcdep.2007.02.004

Zeeb, F. D., \& Winstanley, C. A. (2011). Lesions of the basolateral amygdala and orbitofrontal cortex differentially affect acquisition and performance of a rodent gambling task. The Journal of Neuroscience, 31(6), 2197-2204. doi:10.1523/JNEUROSCI. 5597-10.2011 